\title{
Effect of caffeine on neonatal splanchnic blood flow
}

\author{
Andrew J P Lane, Robert C Coombs, David H Evans, Roy J Levin
}

\begin{abstract}
Doppler ultrasound was used to study the effect of the first intravenous dose of caffeine on splanchnic haemodynamics in preterm neonates. Peak systolic velocity in the superior mesenteric artery and coeliac axis was significantly reduced for 6 hours after caffeine infusion.

The effect of this reduction in blood flow to the neonatal gut is not known.

(Arch Dis Child Fetal Neonatal Ed 1999;80:F128-F129)
\end{abstract}

Keywords: splanchnic circulation; caffeine

The use of vasoactive drugs can influence conditions whose aetiology may be linked to altered states of perfusion, such as intraventricular haemorrhage or necrotising enterocolitis (NEC). Methylxanthines, such as caffeine and its analogues, stimulate the respiratory, vagal, and vasomotor centres in the medulla and are often used to treat apnoea of prematurity. Studies of the cardiovascular effects of the methylxanthines have largely concentrated on the response of the cerebral circulation. ${ }^{1{ }^{2}}$ While Saliba et al could show no effect of caffeine on cerebral blood flow in sick preterm neonates, ${ }^{1}$ Chang and $\mathrm{Gray}^{2}$ demonstrated that aminophylline caused a prolonged and significant reduction in middle and anterior cerebral artery blood flow in preterm human infants which returned to baseline values after about 6 hours.

To date no group has studied the effect of caffeine on the splanchnic blood flow in human preterm neonates although anecdotal reports have linked the xanthines to the occurrence of NEC. ${ }^{3}$ This study was undertaken to define the changes in splanchnic perfusion in preterm human neonates following a loading dose of caffeine.

Department of Neonatology, Jessop Hospital for Women,

Leavygreave Road, Sheffield S3 7RE

A J P Lane

R C Coombs

Department of Biomedical Science, University of Sheffield A J P Lane

R J Levin

Department of Medical Physics, Leicester Royal Infirmary D H Evans

Correspondence to: Dr Robert Coombs.

Accepted 13 August 1998

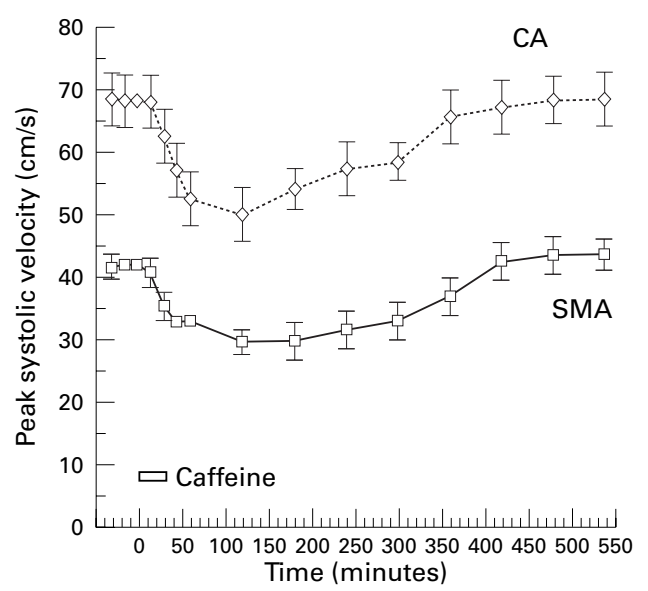

Figure 1 Effect of caffeine (slow intravenous infusion of caffeine citrate, $50 \mathrm{mg} / \mathrm{kg}$ over $30 \mathrm{~min}$ ) on peak systolic velocity in the coeliac axis (CA) and superior mesenteric artery (SMA) in preterm infants. Data are presented as mean (SEM).

Doppler recordings. No fewer than 15 consecutive pulses were accepted for analysis. The velocity measurements were corrected for the angle of insonation which was kept between 0 and 30 degrees.

Each baby was studied at 30 and 15 minutes before caffeine infusion which was then infused over a 30 minute period. The start of the infusion was classed 0 minutes and each baby was restudied at $15,30,60,120,180,240,300$, 360,420 and 480 minutes after caffeine infusion.

\section{Results}

The effect of a slow intravenous infusion of caffeine citrate on peak systolic velocity (PSV) is shown in fig 1. It is important to note that changes in peak systolic velocity reflect changes in mean velocity. There was a significant $(p<0.05)$ and rapid reduction in PSV in both the coeliac axis and superior mesenteric artery which began during the infusion of caffeine and was maximal at (mean (SEM) 176 (24) minutes and 133 (22) minutes, respectively. Blood flow velocity remained depressed for 2 to 3 hours after caffeine infusion, after which there was a slow recovery with a return to baseline after about 6 hours. The maximum percentage decline in coeliac axis and superior mesenteric arterial PSV was not significantly different (29 (3) and 31 (4) \%; p>0.05 using Student's $t$ test). No ill effects were noted in the subjects.

\section{Discussion}

The pharmacological actions of caffeine and theophylline are diverse, and in neonates they are used primarily for their stimulatory effect 
on the respiratory centre. The methylxanthines have been implicated in the pathogenesis of necrotising enterocolitis. ${ }^{3}$ Robinson et a ${ }^{\beta}$ reported five anecdotal cases in neonates who had received oral xanthines. These babies were all very low birthweight and preterm and as such were at risk. But Jones ${ }^{5}$ presented data from her own unit over a three year period which demonstrated no link between either oral or intravenous theophylline and necrotising enterocolitis in a large cohort of 269 babies. Although not significant, those infants who had received xanthines had a slightly higher incidence $(9 \%)$ than those who had received no xanthines $(5 \%)$.

We can only speculate as to the mechanism of this probable vasoconstriction following the first dose of intravenous caffeine. In adults who do not normally take caffeine it induces a decrease in regional blood flow not seen in regular users. ${ }^{6}$ Caffeine affects both the optic and cerebral circulation and inhibits adenosine induced vasodilatation..$^{7-9}$ In vitro there seems to be both endothelium dependent and endothelium independent vasoconstriction with caffeine. ${ }^{10}$ Caffeine seems to have an important role in the regulation of intracellular calcium. At a cellular level there are caffeine and noradrenaline sensitive calcium stores as well as caffeine sensitive but noradrenaline insensitive stores. ${ }^{11}$

Our data show that the first slow intravenous caffeine infusion to preterm neonates causes a prolonged and significant reduction in splanchnic perfusion. We have already shown that a similar slow infusion of indomethacin had no effect on the splanchnic vasculature, ${ }^{4}$ while bolus administration caused a significant re- duction in splanchnic blood flow velocities. However, the response to indomethacin was much more short lived than that after caffeine and had returned to baseline after about one hour.

Whether this observation is of clinical importance is unclear, but it is a finding of which clinicians should be aware and one which deserves further study, as does the nature of the response to the second and subsequent doses.

We thank the special care nursing staff at the Jessop Hospital for Women and the parents of the babies recruited.

1 Saliba E, Autret E, Nasr C, Suc AL, Laugier J. Perinatal pharmacology and cerebral blood flow. Biol Neonate 1992;62:252-7.

2 Chang J, Gray PH. Aminophylline therapy and cerebral blood flow velocity in preterm infants. $\mathscr{f}$ Paediatr Child Health 1994;30:123-5.

3 Robinson MJ, Clayden GS, Smith MF. Xanthines and necrotising enterocolitis. Arch Dis Child 1980;55:494-5.

4 Coombs RC, Morgan MEI, Durbin GM, Booth IW, McNeish AS. Gut blood flow velocities in the newborn: effects of patent ductus arteriosus and parenteral indomethacin. Arch Dis Child 1990;65:1067-71.

5 Jones RAK. Xanthines and necrotising enterocolitis. Arch Dis Child 1981;5:238

6 Casiglia E Paleari CD, Petucco S, et al. Haemodynamic effects of coffee and purified caffeine in normal volunteers: a placebo controlled clinical study $f$ Hypertension 1992;6:95-9.

7 Lotfi K, Grunwald JE. The effect of caffeine on the human macular circulation Invest Ophthalmol Vis Sci macular circul

8 Stubbs TA, Macdonald IA. Systemic and regional effects of caffeine and alcohol in fasting subjects. Clin Auton Res 1995;5:123-7.

9 Smits P, Lenders JW, Thein T. Caffeine and theophylline attenuate adenosine-induced vasodilation in humans. Clin Pharmacol Ther 1990;48:410-18.

10 Jino H, Kurahashi K, Usui H, Shirahase H, Nakata Y, Shimizu Y. Pharmacological natures of caffeine-induced endothelium-dependent and-independent contraction in canine mesenteric artery. Life Sci 1995;57:1155-62.

1 Baro I, Eisner DA. factors controlling changes in intracellular $\mathrm{Ca}_{2}+$ concentration produced by noradrenalin in rat mesenteric artery smooth muscle cells. F Physiol 1995;482:247-58 